\title{
The management and outcome for patients with chronic subdural hematoma: a prospective, multicenter, observational cohort study in the United Kingdom
}

\author{
Paul M. Brennan, FRCS, PhD, ${ }^{1}$ Angelos G. Kolias, MRCS, PhD, ${ }^{2}$ Alexis J. Joannides, MRCS, PhD, ${ }^{2}$ \\ Jonathan Shapey, MRCS, FRCS, ${ }^{3}$ Hani J. Marcus, MRCS, ${ }^{4}$ Barbara A. Gregson, PhD, ${ }^{5}$ \\ Patrick J. Grover, MSc, MA, ${ }^{6}$ Peter J. Hutchinson, FRCS, PhD, ${ }^{2}$ and lan C. Coulter, MRCS, \\ on behalf of the British Neurosurgical Trainee Research Collaborative
}

\begin{abstract}
'Department of Clinical Neurosciences, Western General Hospital, Edinburgh; 'Division of Neurosurgery, Addenbrooke's Hospital and University of Cambridge, Cambridge; ${ }^{3}$ Department of Neurosurgery, National Hospital for Neurology and Neurosurgery, London; ${ }^{4}$ Department of Neurosurgery, Charing Cross Hospital, London; ${ }^{5}$ Neurosurgical Trials Group, Newcastle University, Newcastle upon Tyne; ${ }^{6}$ Department of Neurosurgery, Royal London Hospital, London; and ${ }^{7}$ Department of Neurosurgery, James Cook Hospital, Middleborough, United Kingdom
\end{abstract}

OBJECTIVE Symptomatic chronic subdural hematoma (CSDH) will become an increasingly common presentation in neurosurgical practice as the population ages, but quality evidence is still lacking to guide the optimal management for these patients. The British Neurosurgical Trainee Research Collaborative (BNTRC) was established by neurosurgical trainees in 2012 to improve research by combining the efforts of trainees in each of the United Kingdom (UK) and Ireland's neurosurgical units (NSUs). The authors present the first study by the BNTRC that describes current management and outcomes for patients with CSDH throughout the UK and Ireland. This provides a resource both for current clinical practice and future clinical research on $\mathrm{CSDH}$.

METHODS Data on management and outcomes for patients with CSDH referred to UK and Ireland NSUs were collected prospectively over an 8-month period and audited against criteria predefined from the literature: NSU mortality $<5 \%$, NSU morbidity $<10 \%$, symptomatic recurrence within 60 days requiring repeat surgery $<20 \%$, and unfavorable functional status (modified Rankin Scale score of 4-6) at NSU discharge $<30 \%$.

RESULTS Data from 1205 patients in 26 NSUs were collected. Bur-hole craniostomy was the most common procedure $(89 \%)$, and symptomatic recurrence requiring repeat surgery within 60 days was observed in $9 \%$ of patients. Criteria on mortality $(2 \%)$, rate of recurrence $(9 \%)$, and unfavorable functional outcome $(22 \%)$ were met, but morbidity was greater than expected (14\%). Multivariate analysis demonstrated that failure to insert a drain intraoperatively independently predicted recurrence and unfavorable functional outcome $(p=0.011$ and $p=0.048$, respectively). Increasing patient age ( $p$ $<0.00001)$, postoperative bed rest $(p=0.019)$, and use of a single bur hole $(p=0.020)$ independently predicted unfavorable functional outcomes, but prescription of high-flow oxygen or preoperative use of antiplatelet medications did not.

CONCLUSIONS This is the largest prospective CSDH study and helps establish national standards. It has confirmed in a real-world setting the effectiveness of placing a subdural drain. This study identified a number of modifiable prognostic factors but questions the necessity of some common aspects of CSDH management, such as enforced postoperative bed rest. Future studies should seek to establish how practitioners can optimize perioperative care of patients with CSDH to reduce morbidity as well as minimize CSDH recurrence. The BNTRC is unique worldwide, conducting multicenter trainee-led research and audits. This study demonstrates that collaborative research networks are powerful tools to interrogate clinical research questions.

https://thejns.org/doi/abs/10.3171/2016.8.JNS16134

KEY WORDS chronic subdural hematoma; neurosurgery; outcomes; vascular disorders

ABBREVIATIONS BHC $=$ bur-hole craniostomy; BNTRC = British Neurosurgical Trainee Research Collaborative CSDH $=$ chronic subdural hematoma; GCS $=$ Glasgow Coma Scale; INR = international normalized ratio; mRS = modified Rankin Scale; NSU = neurosurgical unit; ORION = Outcome Registry Intervention and Operation Network; RCT = randomized controlled trial; SBNS = Society of British Neurological Surgeons; TDC = twist-drill craniostomy; UK = United Kingdom.

SUBMITTED January 17, 2016. ACCEPTED August 3, 2016.

INCLUDE WHEN CITING Published online November 11, 2016; DOI: 10.3171/2016.8.JNS16134. 
$\mathrm{C}$ HRONIC subdural hematoma (CSDH) is a collection of liquefied blood between the dura mater and the arachnoid layer of the brain. The incidence is 8.2/100,000/year after 70 years of age. ${ }^{2}$ With an ageing population, a rise in $\mathrm{CSDH}$ prevalence is anticipated. Spontaneous resolution can occur, but surgical evacuation is indicated in patients who deteriorate or do not improve. However, there is little Level I evidence describing optimal surgical and perioperative management strategies.

The 3 most common surgical techniques to treat $\mathrm{CSDH}$ are twist-drill craniostomy (TDC), bur-hole craniostomy (BHC), and craniotomy. Although all 3 techniques have approximately the same mortality rate $(2 \%-4 \%)$, craniotomy has significantly higher morbidity, and TDC has a higher rate of recurrence, suggesting that $\mathrm{BHC}$ is the preferred technique. ${ }^{25}$ In contrast, a more recent meta-analysis concluded that TDC should be the first-line treatment, with craniotomy reserved for symptomatic recurrence. ${ }^{8}$

Other aspects of perioperative and postoperative management may also influence outcome. A randomized controlled trial (RCT) demonstrated that subdural drains left in situ after BHC reduced $\mathrm{CSDH}$ recurrence requiring redrainage $(9.3 \%$ vs $24.0 \%$ recurrence with vs without drain). ${ }^{21}$ The benefit of drains was confirmed in a recent meta-analysis. ${ }^{3}$ However, conflicting evidence for other aspects of CSDH management leads to considerable variation in practice. For example, the optimal number of bur holes (1 vs 2 ) or the benefit of using intraoperative irrigation is uncertain. ${ }^{12,19,23}$ Postoperatively there is contradictory advice about the relative risks and benefits of bed rest versus early mobilization.,15,17 The benefit of corticosteroids as either a primary treatment or an adjunct to surgery remains unclear. ${ }^{6,26}$ Opinions also vary regarding the need to administer agents to mitigate the biological effects of antiplatelet agents (e.g., platelet transfusion for aspirin). Observational studies suggest that antiplatelet and anticoagulant use preoperatively is associated with higher rates of CSDH recurrence. ${ }^{4,9,22}$ Conversely, a retrospective study of 58 patients suggested that early surgery for those taking antiplatelets without drug cessation or platelet infusion may be safe..$^{18}$

Patients with CSDH would benefit from a stronger evidence base for the management of this condition. The present study aims to present the clinical, management, and outcome characteristics for patients with CSDH across the United Kingdom (UK) and Ireland. From these data we aimed to identify variables independently associated with symptomatic CSDH recurrence and unfavorable outcomes that could be interrogated in future studies. This was the first study to be conducted by the British Neurosurgical Trainee Research Collaborative (BNTRC).

\section{Methods}

We conducted a nationwide, multicenter, prospective cohort study to describe the clinical characteristics of patients with $\mathrm{CSDH}$, to assess the variation in operative and perioperative strategies, and to ascertain short-term outcomes evaluated against best-practice criteria as determined from a review of the published literature. The Strengthening the Reporting of Observational Studies in
Epidemiology (STROBE) checklist was used in the preparation of this manuscript. ${ }^{24}$

\section{Participants and Study Settings}

Study participants were identified and enrolled at 26 of the $33 \mathrm{UK}$ and Ireland neurosurgical units (NSUs) between May 2013 and January 2014. Eligibility criteria were age $>16$ years, presentation with a primary or recurrent CSDH confirmed on cranial imaging, and referral to a participating NSU. A CSDH was defined radiologically as a predominantly hypodense, isodense, or mixed-density subdural collection. Patients with other pathologies identified at operation or during subsequent management were excluded (e.g., vascular malformations, subdural empyema). The study protocol was approved by the Academic Committee of the Society of British Neurological Surgeons (SBNS) and has been published previously. ${ }^{7}$ The study was supported by the SBNS and formed part of the Neurosurgical National Audit Program.

\section{Outcome Measures and Audit Standards}

Audit standards were determined from the literature, as described previously: 7 NSU mortality $<5 \%$, NSU morbidity $<10 \%$, symptomatic recurrence within 60 days requiring repeat surgery $<20 \%$, and unfavorable functional status (modified Rankin Scale [mRS] score of 4-6) at NSU discharge $<30 \%$.

\section{Data Collection}

The BNTRC is a network of neurosurgical trainees and supervising consultants in each NSU in the UK and Ireland. Local trainee investigators identified patients at the time of admission to the NSU from on-call referral databases or operating theater logbooks. Patient demographic data, baseline characteristics including medical comorbidities and relevant medication history, and details of pre-, intra-, and postoperative management were collected. A minimum data set including baseline characteristics and proposed management was collected for patients referred to but not transferred into the NSU. Reoperation within 60 days of index admission was identified and recorded. The mRS score at discharge from the NSU, morbidity and mortality in the NSU, destination at discharge from the NSU, and length of stay in the NSU were also recorded.

Data were submitted to a secure online database maintained by the Outcome Registry Intervention and Operation Network (ORION) at the University of Cambridge. The ORION database complies with Department of Health Information Governance policies and with standards for secure processing of patient health care data laid out in the Information Governance Toolkit of the Health and Social Care Information Centre. Each NSU was the data controller for its own data. Local governance approvals were in place in each participating NSU.

\section{Data Analysis}

Anonymized data were collated and checked for errors prior to analysis. Patients with missing data sets were excluded if the missing data were relevant to that particular analysis. Data were analyzed according to the predefined 
TABLE 1. Reasons why 382 patients were not transferred to the NSU

\begin{tabular}{lc}
\hline \multicolumn{1}{c}{ Reason } & $\begin{array}{c}\text { No. of } \\
\text { Patients (\%) }\end{array}$ \\
\hline Small collection \& not sufficient to explain symptoms & $152(40)$ \\
\hline Small collection, patient believed to be asymptomatic & $119(31)$ \\
\hline $\begin{array}{l}\text { Deemed not in best interest due to comorbidities } \\
\text { Receiving antiplatelet or anticoagulation meds-to be } \\
\text { admitted for elective drainage }\end{array}$ & $62(16)$ \\
\hline Deemed futile due to poor status & $18(5)$ \\
\hline Meds = medications.
\end{tabular}

audit standards, using 1-sample tests. Multivariable logistic regression models were used to assess the impact of variables on outcome, and odds ratios are reported. Data were analyzed using IBM SPSS Statistics software, version 21.

\section{Results}

\section{Demographic Data}

Data were collected for 1205 patients with CSDH referred to 26 NSUs; recruitment per unit ranged from 4 to 175 patients (mean 46 patients). Of 1205 patients referred, 823 (68.3\%) were accepted for NSU admission. In the remaining 382 patients, CSDH was managed at their referring hospital; 24 of these patients were subsequently transferred to the NSU, but have been excluded from the outcome analysis because of incomplete data. The most common reason for not being transferred was that the subdural collection was considered small and insufficient to explain a patient's symptoms, or that the patient was asymptomatic (Table 1).

\section{Baseline Clinical Characteristics of Transferred Patients}

The median age of patients who were admitted was 77 years (range 20-99 years). Sixty-eight percent were male, and $62 \%(514 / 823)$ had a documented history of head injury in the preceding 3 months. The demographic and baseline characteristics of patients are listed in Table 2.

Cognitive impairment was the most frequent presenting symptom of transferred patients (58\%), followed by hemiparesis (41\%) and headache (41\%) (Table 2). The majority of patients had a Glasgow Coma Scale (GCS) score of 13-15 (88\%), which was not significantly different from nontransferred patients (89\%). Patients' functional status on admission was determined using the $\mathrm{mRS}$, and the median score was 3.

\section{Management of CSDH in Patients Transferred to an NSU Steroid Use}

Twenty-six patients (3\%) received a course of dexamethasone. Sixteen $(2 \%)$ received the drug as the primary treatment modality for a median of 6.5 days (range 3-21 days), with a median dose of $4 \mathrm{mg}$ per day (range 4-16 mg). In 10 patients (1\%) steroids were administered as adjuvant treatment perioperatively for a median of 6
TABLE 2. Demographic data and baseline characteristics for 823 patients who were transferred to the NSU

\begin{tabular}{|c|c|}
\hline Characteristic & No. of Patients (\%) \\
\hline \multicolumn{2}{|l|}{ Premorbid mobility } \\
\hline Independent & $585(71)$ \\
\hline Walking stick & $151(18)$ \\
\hline Zimmer frame & $75(9)$ \\
\hline Wheelchair & $8(1)$ \\
\hline Bed bound & $4(<1)$ \\
\hline \multicolumn{2}{|l|}{ Premorbid residence } \\
\hline Independent & $637(77)$ \\
\hline Caregiver & $128(16)$ \\
\hline Residential home & $37(4)$ \\
\hline Nursing home & $21(3)$ \\
\hline \multicolumn{2}{|l|}{ Reported medical history } \\
\hline Ischemic heart disease & $208(25)$ \\
\hline Arrhythmia & $172(21)$ \\
\hline Diabetes & $133(16)$ \\
\hline Cerebrovascular disease & $132(16)$ \\
\hline Dementia & $89(11)$ \\
\hline Malignancy & $70(9)$ \\
\hline Chronic obstructive pulmonary disease & $49(6)$ \\
\hline Epilepsy & $35(4)$ \\
\hline Metallic valve & $18(2)$ \\
\hline Shunt & $7(1)$ \\
\hline \multicolumn{2}{|l|}{ Presenting complaint* } \\
\hline Cognitive impairment & $480(58)$ \\
\hline Hemiparesis & $336(41)$ \\
\hline Headache & $336(41)$ \\
\hline Gait disturbance & $264(32)$ \\
\hline Dysphasia & $118(14)$ \\
\hline Incontinence & $39(5)$ \\
\hline Seizure & $30(4)$ \\
\hline Facial droop & $28(3)$ \\
\hline No documented symptoms & $33(4)$ \\
\hline
\end{tabular}

* Individual patients often had multiple medical problems and presenting complaints.

days (range 1-40 days), with a median dose of $8 \mathrm{mg}$ per day (range 4-12 mg). Of the 382 patients who were not transferred, steroid therapy was recommended in 39 cases $(10 \%)$.

\section{Perioperative Clotting}

Of the patients who were transferred, in 356/823 (43\%) anticoagulant or antiplatelet medication was prescribed at the time of referral: 171 received aspirin $(21 \%), 160$ warfarin (19\%), 36 clopidogrel (4\%), 6 dipyridamole (1\%), and 12 got other medications not listed (1\%). Aspirin was discontinued a median of 3 days prior to surgery (range $0-44$ days). Of the patients taking aspirin, 49/171 (28.7\%) received a transfusion of platelets preoperatively. For patients taking warfarin $(n=160)$, the most common reversal strategies were vitamin $\mathrm{K}+$ clotting factors (82), vitamin 
TABLE 3. Perioperative morbidity during NSU admission

\begin{tabular}{lc}
\hline \multicolumn{1}{c}{ Postop Morbidity } & No. of Episodes (\% of patients) \\
\hline Pneumonia & $60(8)$ \\
\hline Neurological deficit & $39(5)$ \\
\hline Seizure & $19(2)$ \\
\hline Arrhythmia & $12(2)$ \\
\hline Stroke & $9(1)$ \\
\hline Surgical site infection & $8(1)$ \\
\hline Myocardial infarction & $4(<1)$ \\
\hline Venous thromboembolism & $4(<1)$ \\
\hline
\end{tabular}

Some patients had $>1$ episode of morbidity recorded (155 episodes experienced by 111 patients).

K alone (28), or clotting factors alone (20). Reversal treatment was not reported in 22 patients (14\%).

\section{Surgical Treatment}

Surgical procedures were performed in 787 of 823 patients (96\%): $76 \%$ by registrars, $21 \%$ by senior house officers, and $3 \%$ by consultants. Most operations were performed after induction of general anesthesia (93\%).

Bur-hole craniostomy was the most frequent procedure (700/787 [89\%]), followed by craniotomy (72/787 [9\%]), and TDC in just 1 case $(<1 \%)$; the remaining 14 patients had a craniectomy, or BHC and craniotomy on different sides. Unilateral BHC was most commonly performed using 2 bur holes (89\%); $10 \%$ had a single bur hole. Bur holes were irrigated intraoperatively to remove the subdural collection in $99 \%$ of cases, and a drain was used in $85 \%$ of cases; the drains were most often sited subdurally (92\%) rather than subgaleally. Drains were left in situ for 48 hours $(68 \%)$ or 24 hours $(28 \%)$.

\section{Postoperative Management}

Postoperative bed rest was prescribed in $61 \%$ of patients after BHC (median 12-24 hours). Only $10 \%$ of patients undergoing $\mathrm{BHC}$ received prescribed high-flow oxygen postoperatively. Postoperative cranial imaging was performed in 380 (48\%) patients after surgery; the investigation was described as routine in $58 \%$.

\section{Patient Outcomes}

Outcomes were determined against the previously described criteria.

\section{Mortality}

The audit standard for all causes of mortality in the NSU was $<5 \%$. The mortality and morbidity data were reported for 798/823 transferred patients. Eighteen of 798 patients $(2 \%)$ died during the study. Causes of death included pneumonia (10), other sepsis (2), and stroke (3).

\section{Morbidity}

The audit standard for all causes of morbidity in the NSU was < $10 \%$. The NSU morbidity rate was $14 \%$ $(111 / 798)$, mainly from respiratory tract infection (8\%) (Table 3).

\section{Symptomatic Recurrence Requiring Surgery}

The audit standard for symptomatic recurrence requiring surgery was a rate of $<20 \%$. Follow-up data were available for all 787 patients who underwent surgery. Recurrence occurred within 60 days of primary surgery in 73 patients (9\%). The median duration of time to reoperation was 12 days (range $0-57$ days). There was no significant difference in recurrence between patients who had BHC or craniotomy $(\mathrm{p}=0.831)$.

We determined variables associated with symptomatic recurrence in the 684 patients who underwent de novo BHC for whom data were available, using logistic regression (Table 4). Sixteen patients for whom the BHC was for a CSDH recurrence were excluded from analysis.

Drain insertion after BHC ( $p=0.011$, OR 0.414$)$ and a higher preoperative GCS score $(p=0.008$, OR 0.859) were predictive of reduced recurrence after controlling for the variables shown in Table 4 . These same variables remained predictive of recurrence when all 772 BHC and craniotomy patients were considered together $(p=0.0003$, OR 0.347 and $\mathrm{p}=0.002$, OR 0.855 , respectively).

We dichotomized the 684 patients treated with $\mathrm{BHC}$ according to whether they had unilateral or bilateral CSDH. One hundred fifty-seven of 202 patients in whom bilateral CSDH was documented underwent bilateral BHC. In patients with unilateral BHC, a higher preoperative GCS

TABLE 4. Factors influencing symptomatic recollection of CSDH requiring repeat surgery within 60 days after de novo BHC

\begin{tabular}{|c|c|c|c|c|}
\hline Variable & No Recurrence, $n=626$ & Recurrence, $n=58$ & OR & $p$ Value \\
\hline Median age in yrs, interquartile range & $77,67-84$ & $80,70-87$ & 1.011 & 0.299 \\
\hline Preop GCS score, range & $14,14-15$ & $14,11.75-15$ & 0.859 & 0.008 \\
\hline On antiplatelet meds* & $24 \%$ & $19 \%$ & 0.673 & 0.266 \\
\hline Mixed-density clot & $49 \%$ & $57 \%$ & 1.351 & 0.293 \\
\hline$>1$ bur hole & $92 \%$ & $90 \%$ & 0.902 & 0.833 \\
\hline Bilat op & $24 \%$ & $26 \%$ & 1.167 & 0.635 \\
\hline Drain inserted & $86 \%$ & $74 \%$ & 0.414 & 0.011 \\
\hline Bed rest & $61 \%$ & $57 \%$ & 0.853 & 0.586 \\
\hline High-flow $\mathrm{O}_{2}$ & $9 \%$ & $9 \%$ & 0.887 & 0.811 \\
\hline
\end{tabular}

Calculated with logistic regression. The ORs are for CSDH recollection. Three patients were not included in logistic regression because their age was missing.

* For the majority of patients in whom anticoagulants were prescribed, the international normalized ratio (INR) was corrected prior to surgery so that INR was not considered as a variable in this logistic regression analysis. 


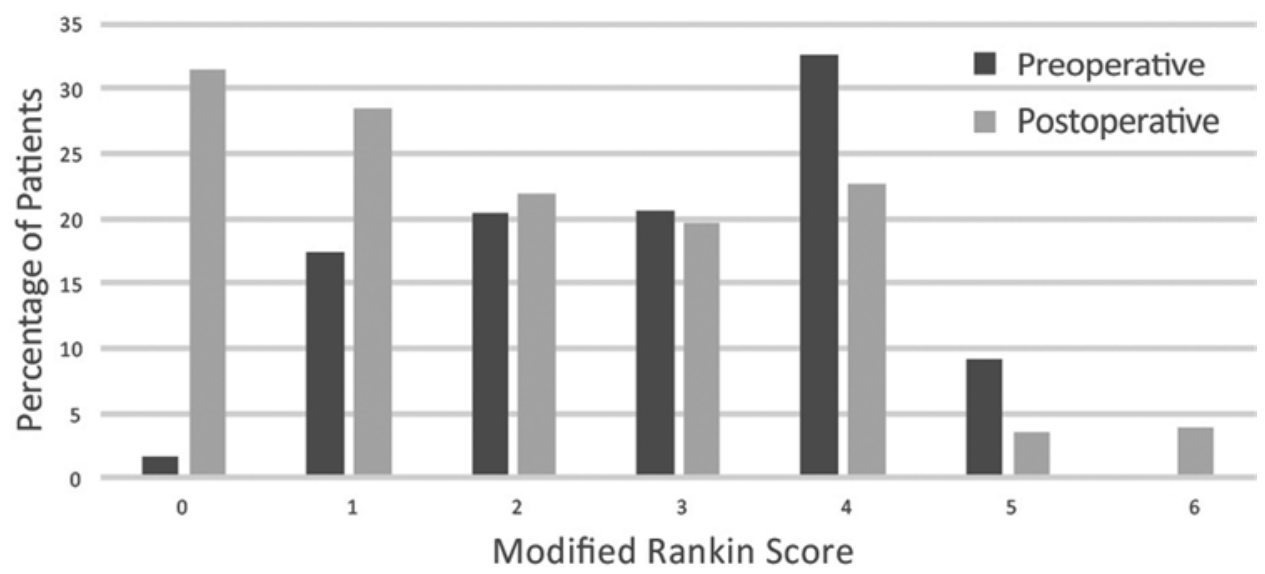

FIG. 1. Bar graph showing a comparison of the proportion of all patients with a given mRS score in the pre- and postoperative patient cohorts. The graph demonstrates the shift to better functional status on discharge compared with status on admission to the NSU.

score $(\mathrm{p}=0.006$, OR 0.839) and drain insertion after BHC $(\mathrm{p}=0.004$, OR 0.317) were still predictive of reduced recurrence. In the group that underwent bilateral BHC, none of the variables reached statistical significance (Supplementary Table 1). Bilateral BHC was not an independent risk factor for recurrence $(\mathrm{p}=0.867)$.

There was no significant difference in recurrence rates between patients having 1 bur hole $(10 \%)$ or multiple bur holes $(8 \%)$ for unilateral CSDH $(\mathrm{p}=0.875)$ when controlling for the other variables. Antiplatelet medication use preoperatively, the administration of high-flow oxygen in the postoperative period, and bed rest did not independently affect recurrence significantly $(p=0.266, p=0.811$, and $\mathrm{p}=0.586$, respectively), even when the BHC cohort was divided into unilateral and bilateral CSDH groups (Table 4, Supplementary Tables 1 and 2). The preoperative appearance on CT imaging of a mixed-density subdural collection, rather than a homogeneously isodense or hypodense clot, did not predict recurrence $(\mathrm{p}=0.293)($ Table 4$)$.

\section{Functional Outcome}

The audit standard for functional outcome was an unfa- vorable mRS score (4-6) at NSU discharge of $<30 \%$. The $\mathrm{mRS}$ score data were available for 798 patients at the point of discharge. An unfavorable mRS score occurred in 176 patients (22\%). The median mRS score was 2, compared with 3 preoperatively (Fig. 1). Factors predicting an unfavorable $\mathrm{mRS}$ score were determined in the 684 patients who had de novo BHC by using logistic regression; 148 of these patients had an unfavorable mRS score (Table 5).

In the BHC group a favorable preoperative mRS score $(\leq 3)$ was independently predictive of a favorable $\mathrm{mRS}$ score at discharge ( $<<0.00001$, OR 2.13), as was drain insertion $(p=0.048)$, good preoperative GCS score ( $p$ $=0.01)$, and lower age $(\mathrm{p}<0.00001)$; the median age of patients with a favorable mRS score was 76 years (range 65-83 years), compared with 84 years (range 76-88 years) for those with an unfavorable mRS score. Prescribed postoperative bed rest predicted a poor outcome $(\mathrm{p}=0.019)$. Interestingly, although the number of bur holes for BHC did not predict recurrence, the use of multiple bur holes did predict a favorable functional outcome $(p=0.020)$. Preoperative midline shift did not significantly correlate with functional outcome $(\mathrm{p}=0.857)$.

The same factors remained significant predictors of

TABLE 5. Factors influencing functional outcome at NSU discharge after de novo BHC

\begin{tabular}{|c|c|c|c|c|}
\hline Variable & Favorable $\mathrm{mRS}$ Score $(0-3), \mathrm{n}=518$ & Unfavorable mRS Score (4-6), $n=148$ & OR & $p$ Value \\
\hline $\begin{array}{l}\text { Median age in yrs, inter- } \\
\text { quartile range }\end{array}$ & $76,65-83$ & $84,76-88$ & 1.074 & $<0.00001$ \\
\hline Initial mRS score, range & $3,2-4$ & $4,3.25-4$ & 2.131 & $<0.00001$ \\
\hline Preop GCS score, range & $14,14-15$ & $14,12-14$ & 0.871 & 0.011 \\
\hline On antiplatelet meds & $20 \%$ & $33 \%$ & 1.326 & 0.236 \\
\hline Mixed-density clot & $49 \%$ & $52 \%$ & 0.805 & 0.329 \\
\hline$>1$ bur hole & $93 \%$ & $86 \%$ & 0.412 & 0.020 \\
\hline Bilat op & $25 \%$ & $23 \%$ & 1.176 & 0.530 \\
\hline Drain inserted & $85 \%$ & $83 \%$ & 0.497 & 0.048 \\
\hline Bed rest & $58 \%$ & $67 \%$ & 1.747 & 0.019 \\
\hline High-flow $\mathrm{O}_{2}$ & $9 \%$ & $9 \%$ & 0.732 & 0.408 \\
\hline
\end{tabular}

Calculated with logistic regression. The ORs are for unfavorable functional outcome. Of 684 patients, in 18 the outcome was not recorded; 3 more were not included in logistic regression because their age was not recorded. 
poor outcome when the patients treated with BHC were considered along with those treated with craniotomy. When patients with unilateral or bilateral CSDH were examined separately, age and initial mRS score remained significant in both groups $(\mathrm{p}<0.0001$ and $\mathrm{p}=0.0018$, respectively; see Supplementary Tables 3 and 4). In the unilateral BHC-only group, bed rest $(\mathrm{p}=0.001$, OR 2.637) and drain insertion ( $\mathrm{p}=0.002$, OR 0.320) increased in significance; having multiple bur holes was unchanged ( $\mathrm{p}$ $=0.030$, OR 0.407); and preoperative GCS score became almost nonsignificant ( $\mathrm{p}=0.054$, OR 0.887).

\section{Length of Stay and Discharge Destination}

The median length of NSU stay was 7 days (range 1-179 days); there was no statistical difference between patients treated with BHC and craniotomy. At NSU discharge, $47 \%$ of patients (358) were transferred to a local hospital for ongoing care, and 49\%-one-sixth of whom required caregivers-were discharged home.

\section{Discussion}

This was the BNTRC's first study and represents the largest prospective, observational, multicenter study of $\mathrm{CSDH}$ management, NSU outcomes, and 60-day recurrence rates. The BNTRC was established by neurosurgical trainees in 2012 to improve research by combining the efforts of trainees in each of the UK and Ireland's NSUs. ${ }^{14}$ The collaborative group provides a semiformal structure to support individuals; a steering group devises the project protocol and invites trainees to join the study as collaborators. Individual trainees in each NSU volunteer as data collectors. Both authorship and collaborator status on publications is defined in advance. Upon project completion the data become available to all collaborating members to permit further analysis.

This study provides valuable insights into current management practices for patients with $\mathrm{CSDH}$. It will inform contemporary practice. It validates in a real-world setting the 2009 RCT that demonstrated the effectiveness of subdural drain insertion. ${ }^{21}$ Other key observations should be validated in prospective trials. The study defines the current standard of care for patients with symptomatic CSDHs as bur-hole drainage with insertion of a subdural drain for 24-48 hours. There was no clear preference for prescribed bed rest or high-flow oxygen in the postoperative period; bed rest was actually associated with unfavorable functional outcome.

Current practice exceeded predefined audit standards for functional outcome at discharge, in-NSU mortality, and 60-day postoperative symptomatic CSDH recurrence. However, the $14 \%$ incidence of postoperative morbidity was greater than the audit standard of $<10 \%$. This may reflect better data collection in our prospective study compared with existing retrospective studies. Interestingly, the previously largest series (retrospective) of surgically treated cases of CSDH reported $19.6 \%$ morbidity. ${ }^{10}$ There is nevertheless an opportunity to optimize care for CSDH patients, many of whom are elderly and have multiple comorbidities. There may be parallels to achieving this in the same way that changes to perioperative care of patients with femural neck fractures reduced mortality in that vul- nerable population. ${ }^{13}$ The BNTRC is therefore collaborating on a prospective study to examine how to optimize perioperative factors in patients with $\mathrm{CSDH}$ to improve the management of their condition.

The decision to proceed with revision surgery was at the discretion of the patient's consultant neurosurgeon, based on clinical symptoms, correlated with imaging. The $9 \%$ rate of symptomatic recurrence we observed at 60 days may have underestimated the true rate if there were late recurrences, but previous studies have suggested that recurrence is most likely within this time frame. ${ }^{16}$ The median time to recurrence in the present study agrees with that reported previously. ${ }^{4}$ Our study demonstrated that only $48 \%$ of surgically treated patients underwent postoperative imaging, so there may have been incidents of recurrence that did not reach clinical significance but would have been detected radiologically. Because the need for redo surgery is based on symptomatic recurrence, radiological recurrence alone is of less importance.

We observed that the number of bur holes was not an independent risk factor for $\mathrm{CSDH}$ recurrence after $\mathrm{BHC}$, but single bur holes were associated with worse functional outcomes at discharge. Preoperative mRS score was not predictive of the number of bur holes used in BHC, and the reason for the worse functional outcomes is not clear. Previous retrospective studies have suggested that a single bur hole is as effective as 2 in selected cases. ${ }^{11,12}$ Future studies should interrogate this further. A single bur hole may be preferred if associated with reduced operative length and in turn lower postoperative morbidity, but this has yet to be demonstrated.

Multiple bur holes may traditionally be preferred where there is a mixed-density subdural collection (mixed between hyperdense and isodense or hypodense material). This is because the hyperdense material represents more acute blood that is thicker than isodense or hypodense blood and does not discharge as easily from a single bur hole. However, our study identified that the radiological appearance of the subdural collection was not predictive of symptomatic recurrence. This may be because the goal of surgery is to reduce the mass effect from the CSDH, not necessarily to remove the hematoma altogether; the residual subdural collection can resolve spontaneously. A single bur hole may be adequate to achieve this, and, interestingly, we observed no correlation between subdural density and the number of bur holes used for BHC.

Evidence for the benefit of corticosteroids in $\mathrm{CSDH}$ management remains scarce. Our study documented steroid use in only a small proportion of patients. The effect of dexamethasone on reduction of the reoperation rate for $\mathrm{CSDH}$ is the focus of several ongoing studies, including an RCT comparing dexamethasone to placebo after BHC, a study supported by the BNTRC (dexcsdh.org).

Anticoagulant and antiplatelet use have been implicated in both the development and recurrence of CSDH. ${ }^{5}$ In our study, $43 \%$ of the patients transferred to an NSU for treatment were taking an antiplatelet or anticoagulant medication. Strategies for the preoperative optimization of platelet function and coagulation in these patients varied greatly, but the preoperative prescription of antiplatelet agents was not an independent risk factor for recurrence. The impact of anticoagulant agents was not examined, be- 
cause all patients receiving these agents had their international normalized ratio (INR) corrected preoperatively. Although the use of antiplatelet agents was not observed to be associated with recurrence, the heterogeneity in timing of the discontinuation of medication and of reversal strategies suggests that this observation should be interpreted cautiously. Nevertheless, it remains feasible that some patients may not need to discontinue their medication for the traditional 7 days before bur-hole surgery. This could be important, because delay in recommencing these therapies is associated with thromboembolic risk, and the delay of surgery while managing anticoagulant and antiplatelet therapies may also be associated with increased morbidity.

Although our observations are based on data from the UK and Ireland, the results will be of interest internationally because CSDH is common worldwide. There will undoubtedly be variations between countries in the preference for some aspects of CSDH management that were not well represented in UK practice, such as prescription of steroids or use of TDC. The design of prospective studies to develop an evidence base for CSDH management should include all these factors.

There are some limitations to our study. For example, we captured data from patients whose CSDH was managed in NSUs, so we do not know the natural history of patients with CSDH that was managed in hospitals without NSUs. We examined relatively short-term outcome measures, and longer-term outcome measures such as 6-month functional status and mortality rate would be valuable. Our patient cohort was skewed to management with BHC, so we could not make a meaningful analysis of variables predicting outcome in the craniotomy subgroup. In addition, our study demonstrated that surgical drainage of CSDH is performed almost exclusively by trainee neurosurgeons and senior house officers. This is clearly standard practice in the UK, and a previous study has found no association between seniority of the surgeon and postoperative recurrence of CSDH. ${ }^{20}$ It should also be noted that it is our experience that a consultant neurosurgeon is always the person to make a decision to proceed to surgery and is available if needed. The question may nevertheless be raised as to whether patient outcomes would be different if consultant surgeons were the primary surgeon. This is an important question for future study.

\section{Conclusions}

We have defined the preferred strategy for treating symptomatic CSDH as bur-hole drainage with a postoperative closed drainage system. We have observed that many variations in practice do not significantly impact outcome. Further research is required to examine these relationships in more detail and to determine whether standardization of management can improve patient outcomes.

\section{Acknowledgments}

Funding was received from the SBNS. Mr. Hutchinson was supported by a National Institute for Health Research (NIHR) Research Professorship and the NIHR Cambridge Biomedical Research Centre. The study was supported by the Royal College of Surgeons of England (PJH specialty lead for neurosurgery). Mr. N. Mukerji assisted with analyzing the data. We also thank the academic committee of the SBNS for support in the design and conduct of the study.

\section{Appendix \\ Collaborators}

Afshari FT, Ahmed AI, Alli S, Al-Mahfoudh R, Bal J, Belli A, Borg A, Bulters D, Carleton-Bland N, Chari A, Coope D, Cowie CJ, Critchley G, Dambatta S, D'Aquino D, Dhamija B, Dobson G, Fam MD, Glancz L, Gray WP, Halliday J, Hamdan A, Hill CS, Jamjoom AAB, Jones TL, Joshi SM, Kailaya-Vasan A, Karavasili V, Khan SA, King AT, Kuenzel A, Livermore LJ, Lo W, Martin J, Matloob S, Mitchell P, Mowle D, Narayanamurthy H, Nelson RJ, Ngoga D, Noorani I, O'Reilly G, Othman H, Owusu-Agyemang K, Manjunath Prasad KS, Plaha P, Pollock J, Prasad KS, Price R, Pringle C, Ray A, Reaper J, Scotton W, Simms N, Smith S, Statham P, Steele L, St George J, Stovell MG, Tarnaris A, Teo M, Thomson S, Thorne L, Vintu M, Whitfield P, Wilson M, Wilby M, Woodfield J, Zaben M.

\section{References}

1. Abouzari M, Rashidi A, Rezaii J, Esfandiari K, Asadollahi $\mathrm{M}$, Aleali $\mathrm{H}$, et al: The role of postoperative patient posture in the recurrence of traumatic chronic subdural hematoma after burr-hole surgery. Neurosurgery 61:794-797, 2007

2. Adhiyaman V, Asghar M, Ganeshram KN, Bhowmick BK: Chronic subdural haematoma in the elderly. Postgrad Med J 78:71-75, 2002

3. Alcalá-Cerra G, Young AMH, Moscote-Salazar LR, Paternina-Caicedo A: Efficacy and safety of subdural drains after burr-hole evacuation of chronic subdural hematomas: systematic review and meta-analysis of randomized controlled trials. World Neurosurg 82:1148-1157, 2014

4. Baechli H, Nordmann A, Bucher HC, Gratzl O: Demographics and prevalent risk factors of chronic subdural haematoma: results of a large single-center cohort study. Neurosurg Rev 27:263-266, 2004

5. Bakheet MF, Pearce LA, Hart RG: Effect of addition of clopidogrel to aspirin on subdural hematoma: meta-analysis of randomized clinical trials. Int J Stroke 10:501-505, 2015

6. Berghauser Pont LME, Dirven CMF, Dippel DWJ, Verweij $\mathrm{BH}$, Dammers R: The role of corticosteroids in the management of chronic subdural hematoma: a systematic review. Eur J Neurol 19:1397-1403, 2012

7. Coulter IC, Kolias AG, Marcus HJ, Ahmed AI, Alli S, AlMahfoudh R, et al: Proposal for a prospective multi-centre audit of chronic subdural haematoma management in the United Kingdom and Ireland. Br J Neurosurg 28:199-203, 2014

8. Ducruet AF, Grobelny BT, Zacharia BE, Hickman ZL, DeRosa PL, Andersen KN, et al: The surgical management of chronic subdural hematoma. Neurosurg Rev 35:155-169, 2012 (Erratum in Neurosurg Rev 38:771, 2015)

9. Forster MT, Mathé AK, Senft C, Scharrer I, Seifert V, Gerlach R: The influence of preoperative anticoagulation on outcome and quality of life after surgical treatment of chronic subdural hematoma. J Clin Neurosci 17:975-979, 2010

10. Gelabert-González M, Iglesias-Pais M, García-Allut A, Martínez-Rumbo R: Chronic subdural haematoma: surgical treatment and outcome in 1000 cases. Clin Neurol Neurosurg 107:223-229, 2005

11. Han HJ, Park CW, Kim EY, Yoo CJ, Kim YB, Kim WK: One vs. two burr hole craniostomy in surgical treatment of chronic subdural hematoma. J Korean Neurosurg Soc 46:87-92, 2009

12. Kansal R, Nadkarni T, Goel A: Single versus double burr hole drainage of chronic subdural hematomas. A study of 267 cases. J Clin Neurosci 17:428-429, 2010 
13. Kenzora JE, McCarthy RE, Lowell JD, Sledge CB: Hip fracture mortality. Relation to age, treatment, preoperative illness, time of surgery, and complications. Clin Orthop Relat Res (186): 45-56, 1984

14. Kolias AG, Jones TL, Cowie CJ, Coulter IC, Afshari FT, Tarnaris A, et al: A report from the inaugural meeting of the British Neurosurgical Trainee Research Collaborative held in the Royal College of Surgeons of England, 19 October 2012. Br J Neurosurg 27:307-310, 2013

15. Kurabe S, Ozawa T, Watanabe T, Aiba T: Efficacy and safety of postoperative early mobilization for chronic subdural hematoma in elderly patients. Acta Neurochir (Wien) 152:1171-1174, 2010

16. Mori K, Maeda M: Surgical treatment of chronic subdural hematoma in 500 consecutive cases: clinical characteristics, surgical outcome, complications, and recurrence rate. Neurol Med Chir (Tokyo) 41:371-381, 2001

17. Nakajima H, Yasui T, Nishikawa M, Kishi H, Kan M: The role of postoperative patient posture in the recurrence of chronic subdural hematoma: a prospective randomized trial. Surg Neurol 58:385-387, 2002

18. Okano A, Oya S, Fujisawa N, Tsuchiya T, Indo M, Nakamura $\mathrm{T}$, et al: Analysis of risk factors for chronic subdural haematoma recurrence after burr hole surgery: optimal management of patients on antiplatelet therapy. Br J Neurosurg 28:204-208, 2014

19. Pahatouridis D, Alexiou GA, Fotakopoulos G, Mihos E, Zigouris A, Drosos D, et al: Chronic subdural haematomas: a comparative study of an enlarged single burr hole versus double burr hole drainage. Neurosurg Rev 36:151-155, 2013

20. Phang I, Sivakumaran R, Papadopoulos MC: No association between seniority of surgeon and postoperative recurrence of chronic subdural haematoma. Ann R Coll Surg Engl 97:584-588, 2015

21. Santarius T, Kirkpatrick PJ, Ganesan D, Chia HL, Jalloh I, Smielewski P, et al: Use of drains versus no drains after burr-hole evacuation of chronic subdural haematoma: a randomised controlled trial. Lancet 374:1067-1073, 2009

22. Sim YW, Min KS, Lee MS, Kim YG, Kim DH: Recent changes in risk factors of chronic subdural hematoma. J Korean Neurosurg Soc 52:234-239, 2012

23. Taussky P, Fandino J, Landolt H: Number of burr holes as independent predictor of postoperative recurrence in chronic subdural haematoma. Br J Neurosurg 22:279-282, 2008

24. Vandenbroucke JP, von Elm E, Altman DG, Gøtzsche PC, Mulrow CD, Pocock SJ, et al: Strengthening the Reporting of Observational Studies in Epidemiology (STROBE): explanation and elaboration. PLoS Med 4:e297, 2007
25. Weigel R, Schmiedek P, Krauss JK: Outcome of contemporary surgery for chronic subdural haematoma: evidence based review. J Neurol Neurosurg Psychiatry 74:937-943, 2003

26. Zarkou S, Aguilar MI, Patel NP, Wellik KE, Wingerchuk DM, Demaerschalk BM: The role of corticosteroids in the management of chronic subdural hematomas: a critically appraised topic. Neurologist 15:299-302, 2009

\section{Disclosures}

The authors report no conflict of interest concerning the materials or methods used in this study or the findings specified in this paper.

\section{Author Contributions}

Conception and design: Brennan, Kolias, Gregson, Hutchinson, Coulter. Acquisition of data: Brennan, Kolias, Joannides, Shapey, Marcus, Grover, Coulter. Analysis and interpretation of data: Brennan, Joannides, Shapey, Marcus, Coulter. Drafting the article: Brennan, Shapey, Marcus, Coulter. Critically revising the article: Brennan, Kolias, Shapey, Marcus, Hutchinson, Coulter. Reviewed submitted version of manuscript: Brennan, Kolias, Joannides, Shapey, Marcus, Grover, Hutchinson, Coulter. Approved the final version of the manuscript on behalf of all authors: Brennan. Statistical analysis: Gregson, Coulter. Administrative/technical/material support: Brennan. Study supervision: Brennan, Coulter.

\section{Supplemental Information \\ Online-Only Content}

Supplemental material is available with the online version of the article.

Supplementary Tables 1-4. https://thejns.org/doi/suppl/ 10.3171/2016.8.JNS16134.

\section{Previous Presentations}

Portions of this work were presented in abstract form as proceedings at the EANS annual meeting in Prague in 2015 and at the Society of British Neurosurgeons biannual meeting in Southampton in 2015 .

\section{Correspondence}

Paul M. Brennan, Department of Neurosurgery, Western General Hospital, Crewe Rd. South, Edinburgh EH4 2XU, United Kingdom. email: paul.brennan@ed.ac.uk. 